\title{
Magnetic Ordering in Hexagonally Bonded Sheets with First-Row Elements
}

\author{
Susumu Okada and Atsushi Oshiyama \\ Institute of Physics, University of Tsukuba, 1-1-1 Tennodai, Tsukuba 305-8571, Japan \\ and Center for Computational Physics, University of Tsukuba, 1-1-1 Tennodai, Tsukuba 305-8577, Japan
}

(Received 4 April 2001; published 18 September 2001)

\begin{abstract}
We report first-principles total-energy electronic-structure calculations in the density-functional theory performed for hexagonally bonded honeycomb sheets consisting of $\mathrm{B}, \mathrm{N}$, and $\mathrm{C}$ atoms. We find that the ground state of BNC sheets with particular stoichiometry is ferromagnetic. Detailed analyses of energy bands and spin densities unequivocally reveal the nature of the ferromagnetic ordering, leading to an argument that the $\mathrm{BNC}$ sheet is a manifestation of the flat-band ferromagnetism.
\end{abstract}

DOI: $10.1103 /$ PhysRevLett.87.146803

Carbon nanotubes [1] and fullerenes [2] have provided a new paradigm of condensed matters: Local atomic structures are common in the constituent units (threefold coordinated $\mathrm{C}$ atoms generating hexagonal or pentagonal rings), but differences in global network produce a rich variety of properties [3-5] ranging from semiconductors, metals, and superconductors under certain conditions [6,7]. Other first-row elements such as $\mathrm{B}$ and $\mathrm{N}$ are also utilized along with $\mathrm{C}$ to synthesize nanotubes or nanoflakes [8-10], enriching variation in electronic properties [11-13]. Magnetism of these new materials has been less pursued, however: In the early stages of research on fullerenes, a possible magnetic ordering was found in organicmolecule doped $\mathrm{C}_{60}$ [14]; yet the origin of the ordering is unclear.

The Fermi-level density of states $E_{\mathrm{F}}$ and electron correlation are two major factors that induce the ferromagnetic ordering in itinerant electron systems. Each role, along with effects of orbital degeneracy and Van Hove singularity inherent to specific lattices, has been examined for more than a half century with several model Hamiltonians [15] or for real materials [16]. Mechanisms that have been clarified to induce the ferromagnetic ordering are not many, however. The flat-band ferromagnetism is one of such mechanisms which have been proved exactly in the Hubbard model under certain conditions [17-19]: For several specific lattice structures, a delicate balance of transfer integrals results in an energy band which lacks dispersion; the introduction of Hubbard $U$ between the band electrons induces the ferromagnetic ordering. Yet it is uncertain whether the flat-band ferromagnetism is real or fictitious in nature.

The existence of flat bands has been demonstrated in graphite flakes [20]: When the flakes have edges with zigzag shapes, electron states emerge that are localized near but extended along the edges, are located at $E_{\mathrm{F}}$ in energy, and lack dispersion along the edge directions in a part of the Brillouin zone (BZ). The flat bands are not peculiar to $\mathrm{C}$ atoms but come up in hexagonally bonded heterosheets which have borders with zigzag shapes between chemically different elements [13]. An analysis [13,20] shows that the flat-band states (called edge states or border
PACS numbers: 73.21.-b, 71.15.Nc, 75.70.Cn

states) result from a delicate balance of electron transfers among $\pi$ orbitals situated near edge atoms.

In this Letter, we performed total-energy electronicstructure calculations using the density-functional theory (DFT) for hexagonally bonded honeycomb sheets consisting of $\mathrm{B}, \mathrm{C}$, and $\mathrm{N}$ atoms. The calculated total energies, spin densities, and energy bands reveal that the flat-band ferromagnetism is realized in these heterosheets, consisting solely of nonmagnetic first-row elements.

All calculations have been performed by DFT [21,22]. Exchange-correlation energy of interacting electrons is treated in local spin density approximation (LSDA) with a functional form fitted to the Ceperley-Alder result [23,24]. Norm-conserving pseudopotentials generated by using the Troullier-Martins scheme are adopted to describe the electron-ion interaction $[25,26]$. The valence wave functions are expanded by the plane-wave basis set with a cutoff energy of $50 \mathrm{Ry}$ which gives enough convergence of relative total energies of carbon-related materials [25] and of $h$-BN [13]. We adopt the conjugate-gradient minimization scheme for both the electronic-structure calculation and for the geometry optimization [27]. Structural optimization has been performed until the remaining forces are less than $5 \mathrm{~m} \mathrm{Ry} / \AA$. We use a repeating sheet model in which each atomic sheet is separated by $9.0 \AA$ to simulate an isolated honeycomb sheet. Integration over the $\mathrm{BZ}$ is carried out using equidistant $k$-point sampling in which the $k$-point density is equivalent to the case of the 96 point sampling in the conventional $\mathrm{BZ}$ of the graphite monolayer.

Figure 1 shows calculated spin density $n_{\uparrow}(\boldsymbol{r})-n_{\downarrow}(\boldsymbol{r})$ of a graphite flake which has straight zigzag edges. As explained above, there are flat-band states at $E_{\mathrm{F}}$ in the graphite flake with zigzag edges. It is thus expected that a certain magnetic ordering appears on the graphite flakes. The present LSDA calculations have clearly uncovered the existence of the magnetic ordering that is mainly located along the zigzag edges and decays gradually inside. It is not the ferromagnetic ordering, however. There are two atomic sites in its hexagonal primitive cell of graphite and all the atomic sites are classified into two sublattices $A$ and $B$ (bipartite lattice). The number of $\mathrm{C}$ atoms in each lattice 
(a)
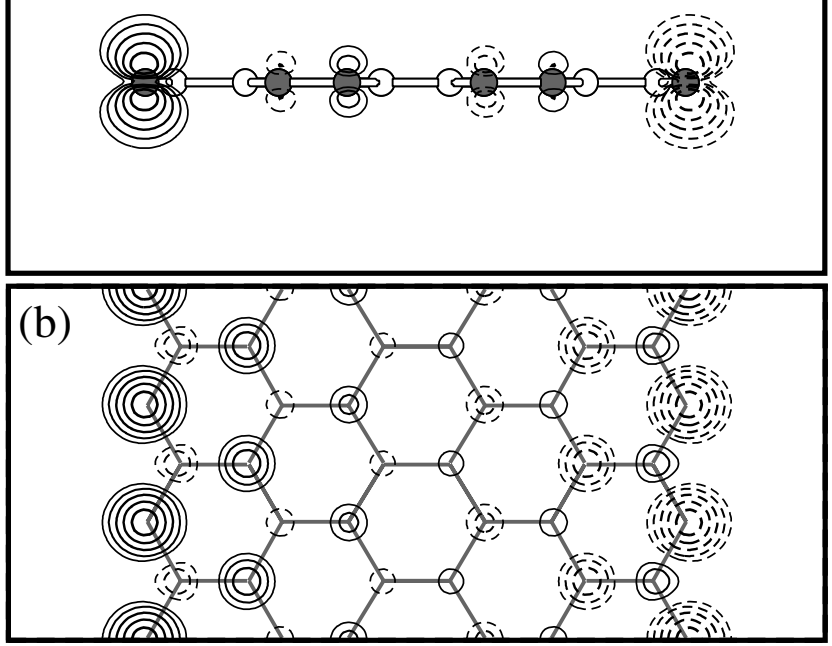

FIG. 1. Contour plots of spin density $n_{\uparrow}(\boldsymbol{r})-n_{\downarrow}(\boldsymbol{r})$ (a) on a plane perpendicular to a graphite flake with zigzag edges and (b) on a plane including the graphite flake. In (a) the edges are perpendicular to the plane and $\mathrm{C}$ atoms on the plane are depicted by shaded circles. Positive and negative values of the spin density are shown by solid and dashed lines, respectively. Each contour represents twice (or half) the density of the adjacent contour lines.

of the flake is identical $\left(N_{A}=N_{B}\right)$. It is clearly observed in Fig. 1 that the spin is polarized in one direction at one sublattice and it is in an opposite direction at the other. As a result, the total spin $S$ of the graphite flakes is vanishing. This corresponds to a theorem $S=\left(N_{A}-N_{B}\right) / 2$ in the bipartite lattice which is proved in the Hubbard model under some conditions [17].

Spin polarization that we found for the graphite flake with zigzag edges is not limited to carbon-atom sheets. This is because the flat-band states responsible for the magnetic ordering are also generated when the borders with the zigzag shape are introduced in hexagonally bonded honeycomb sheets [13]. We therefore consider honeycomb sheets consisting of B, N, and C atoms shown in Figs. 2(a) and 2(b). In these heterosheets, graphite ribbons are separated from each other intervened by honeycomb structures consisting of B-N bonds, thereby producing zigzag borders; the borders are undulating and thus the number of $\mathrm{C}$ atoms belonging to each sublattice is different. In the heterosheet shown in Fig. 2(a) (labeled BNC-I hereafter), a unit cell contains $13 \mathrm{~B}$ and $14 \mathrm{~N}$ atoms, and $21 \mathrm{C}$ atoms which are grouped into $A$-sublattice and $B$-sublattice $\mathrm{C}$ atoms $\left(N_{A}=11\right.$ and $\left.N_{B}=10\right)$. Similarly, in the heterosheet shown in Fig. 2(b) (labeled BNC-II), a unit cell contains $11 \mathrm{~B}$ and $13 \mathrm{~N}$ atoms, and $24 \mathrm{C}$ atoms which are grouped into $N_{A}=13$ and $N_{B}=11 \mathrm{C}$ atoms.

These heterosheets which we introduced may look like artifacts. The existence of the sheets in nature is likely, however. First, the honeycomb structures consisting of
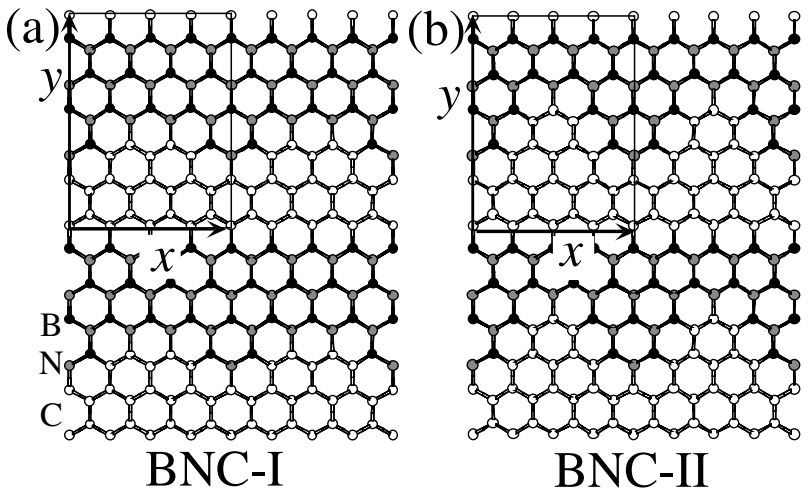

FIG. 2. Top views of fully optimized BNC heterosheets, (a) BNC-I and (b) BNC-II. White, shaded, and black circles denote $\mathrm{C}, \mathrm{B}$, and $\mathrm{N}$ atoms, respectively. The rectangle in each figure denotes the unit cell.

$\mathrm{B}, \mathrm{N}$, and $\mathrm{C}$ atoms have been observed indeed [8-10]. Second, the phase separation of graphite and BN regions leading to the striped structures above is energetically favorable. In fact, we have performed the total-energy calculations for graphite, $\mathrm{BN}, \mathrm{BC}$, and $\mathrm{NC}$ heterosheets by DFT. The calculated bond energies of $\mathrm{B}-\mathrm{C}$ and $\mathrm{N}-\mathrm{C}$ are smaller than that of graphite by 1.52 and $0.81 \mathrm{eV}$, respectively. On the other hand, the bond energy of B-N is smaller than that of graphite only by $0.31 \mathrm{eV}$. Third, undulation of zigzag borders is naturally formed during syntheses of BNC sheets.

For those representatives of honeycomb heterosheets consisting of $\mathrm{B}, \mathrm{N}$, and $\mathrm{C}$ atoms, BNC-I and BNC-II, we found both ferromagnetic and nonmagnetic states as solutions of the Kohn-Sham (KS) equations in the LSDA. The ferromagnetic state is lower in total energy than the nonmagnetic state: The total-energy differences per unit cell are 20 and $11 \mathrm{meV}$ for BNC-I and BNC-II, respectively. This clearly indicates the occurrence of the ferromagnetic ordering in the $\mathrm{BNC}$ heterosheets. The calculated values of the polarized spin per unit cell are 1 and 2 for BNC-I and BNC-II, respectively.

Figure 3 shows calculated energy bands (Kohn-Sham energy levels) of BNC-I and BNC-II in their ferromagnetic states. We observe a single flat-band state in BNC-I and two flat-band states in BNC-II. For these flat-band states, the dispersions even along the $x$ direction (parallel to the border direction) are relatively small, a few tenths of $\mathrm{eV}$. It is of interest that the number of the flat-band states appearing in the BNC heterosheets corresponds to the difference in numbers, $N_{A}-N_{B}$, between $A$-sublattice and $B$-sublattice carbon atoms. The flat-band states for majority (MJ) and minority (MN) spins split by $0.3-0.4 \mathrm{eV}$ for BNC-I and BNC-II (Fig. 3). In the present $k$-point sampling and even in the doubly increased $k$-point sampling in the self-consistent-field (SCF) calculations, the flat-band states for the MJ spin are completely occupied, whereas those for the MN spin are empty. Semiconducting ferromagnetic states have been thus obtained as ground states. 

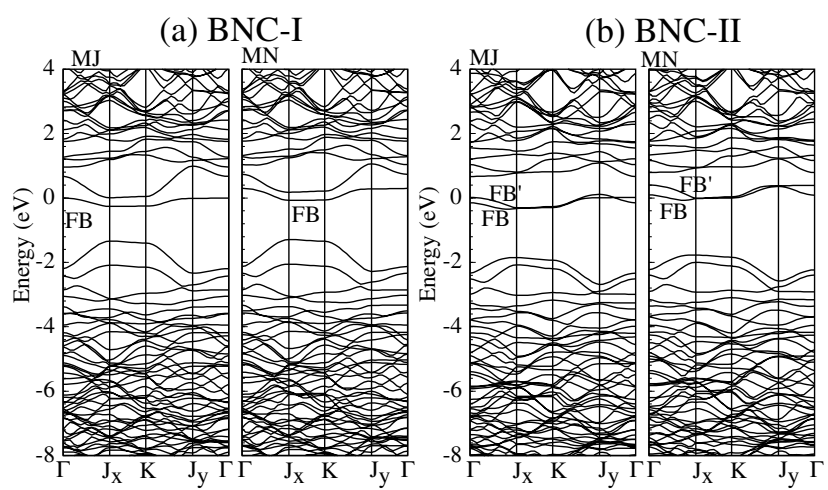

FIG. 3. Energy bands (Kohn-Sham energy levels) of (a) BNC-I and (b) BNC-II for majority (MJ) and minority (MN) spins along the symmetry lines. The directions $\Gamma-J_{x}$ and $\mathrm{K}-J_{y}$ are parallel to $x$ in Fig. 2, whereas the directions $J_{x}-\mathrm{K}$ and $J_{y}-\Gamma$ are parallel to $y$. Flat-band states are labeled by $\mathrm{FB}$ or $\mathrm{FB}^{\prime}$. The Fermi energy is located at $E_{\mathrm{F}}=0$. The MJ- and the MN-spin levels are split completely in the SCF calculation (see text), though they look overlapped along the symmetry lines.

Analyses of KS orbitals of the flat-band states (FB and $\mathrm{FB}^{\prime}$ in Fig. 3) clarify their characteristics. First they consist of $\pi$ orbitals of constituent atoms. Second, they are localized in the border regions of graphite and BN, but at the same time extended along the borders. Yet this peculiar nature is only for a part of BZ: The flat-band states have such characters only near the BZ center, whereas the orbitals for $\mathrm{FB}$ and $\mathrm{FB}^{\prime}$ near the $\mathrm{BZ}$ boundaries are extended over the whole sheet.

Figure 4 shows calculated spin density $n_{\uparrow}(\boldsymbol{r})-n_{\downarrow}(\boldsymbol{r})$ (the up spin is the majority) of BNC-I and BNC-II. It is clear that the majority spin is distributed exclusively on the sublattice $A$ of graphite, whereas the minority spin is on the sublattice $B$. The calculated total spin is identical to $S=\left(N_{A}-N_{B}\right) / 2$ for both BNC-I and BNC-II. This (a) BNC-I

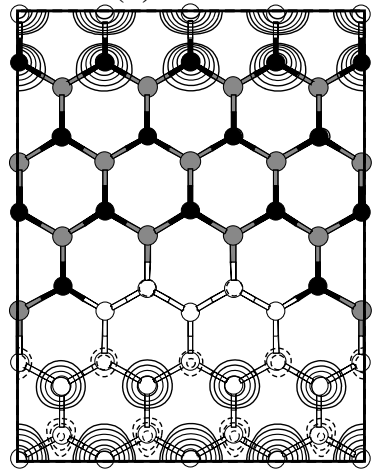

(b) BNC-II

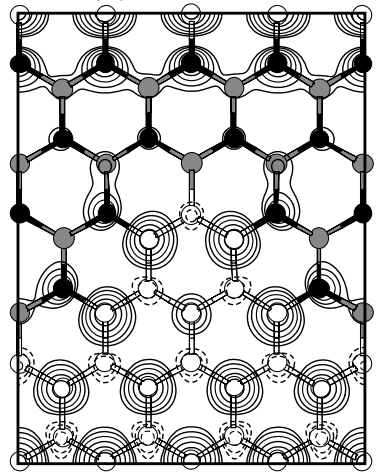

FIG. 4. Contour plot of spin density $n_{\uparrow}(\boldsymbol{r})-n_{\uparrow}(\boldsymbol{r})$ on the BNC heterosheet. (a) BNC-I and (b) BNC-II. Positive and negative values of the spin density are shown by solid and dashed lines, respectively. White, shaded, and black circles denote positions of $\mathrm{C}, \mathrm{B}$, and $\mathrm{N}$ atoms, respectively. Each contour represents twice (or half) the density of its neighboring contour lines. The lowest contour represents $5.27 \times 10^{-3} e / \AA^{3}$. indicates that there is ferrimagnetic ordering in flat bands in the bipartite lattice. BNC-I and BNC-II have periodic structures along the borders. This is unnecessary, however, to realize the ferro- (ferri-) magnetic ordering. The zigzag borders between chemically different elements and the imbalance between the numbers of carbon sublattice sites suffice.

The calculated spin density in BNC-I and BNC-II is distributed on the whole graphite region. This is because the graphite region is so small that the borders occupy a substantial portion of the region. When we prepare larger graphite regions, the spin density is distributed only near the borders. On the other hand, the BN honeycomb region works as a separator between the spin densities. The hexagonal $\mathrm{BN}$ sheet has a large energy gap. Hence the electron states around the Fermi level in the BNC sheets mainly consist of the $\pi$ states of $\mathrm{C}$ atoms. This results in the spin density mainly located in the graphite region. In order to make the ferromagnetic ordering stable, there may be an optimum width of the BN honeycomb region. This situation opens a possibility of spin density engineering in the BNC honeycomb sheet.

In order to further clarify the nature of the ferromagnetic ordering in BNC-I and BNC-II, we consider another honeycomb structure BNC-III [Fig. 5(a)] where the BN region intervenes with the graphite region of BNC-I and the resulting triangular graphite regions are separated from each other. In BNC-III, we have obtained only nonmagnetic states using LSDA. The energy bands (KS energy levels) shown in Fig. 5(b) clearly exhibit the existence of two flat bands. This corresponds to the difference in sublattice sites, $N_{B}-N_{A}=2$ in this case. The energy dispersions of the flat bands are $0.2 \mathrm{eV}$, smaller than those in BNC-II, and the corresponding KS orbitals are distributed near the borders. The situation in BNC-III is similar to that in BNC-I or BNC-II. The ferromagnetic ordering is not realized, however. This is due to the weak coupling between polarized spins located in the triangular graphite (a)

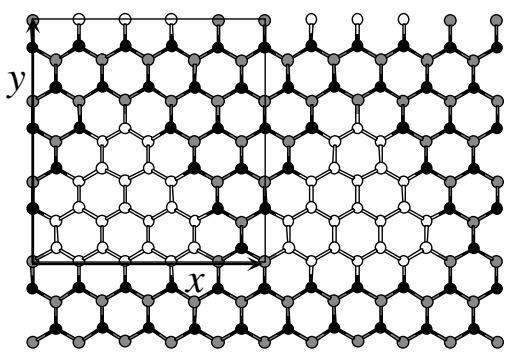

(b)

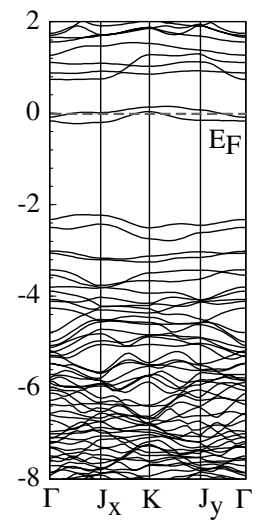

FIG. 5. (a) Top view and (b) band structures of the BNC-III heterosheet. White, shaded, and black circles denote $\mathrm{C}, \mathrm{B}$, and $\mathrm{N}$ atoms, respectively. 
regions. In this case we expect antiferromagnetic coupling between the spins. In fact, further LSDA calculations for BNC-III using double-periodicity unit cells have revealed that the total energy for the antiferromagnetic state is lower than that of the nonmagnetic state by $20 \mathrm{meV}$ per unit cell. This finding in turn corroborates our argument that the ferromagnetic ordering in BNC-I and BNC-II is categorized into the flat-band ferromagnetism.

It is of importance to examine effects of carrier doping on the ferromagnetic ordering in BNC-I and BNC-II. We dope electrons in the flat bands which are empty in Fig. 3. Upon carrier doping in the flat bands, the nonmagnetic states become relatively favorable. Our LSDA calculations show that the transition from the ferromagnetic to the nonmagnetic states occurs at $0.02 e$ and $0.05 e$ per unit cell for BNC-I and BNC-II, respectively. This is the quantification by DFT for the robustness of the flat-band ferromagnetism against carrier doping, which has been discussed only in the Hubbard model previously [19].

In summary, we have found the ferromagnetic ordering for hexagonally bonded honeycomb sheets, consisting solely of the first-row elements. The finding indicates that the heterosheets are examples of the flat-band ferromagnetism in nature and also candidates for the nanometer scale ferromagnet.

We benefited from conversations with K. Kusakabe, K. Nakada, D. S. Hirashima, T. Momoi, and K. Shiraishi. This work was supported in part by JSPS under Contract No. RFTF96P00203, and The Grants-in-Aid No. 11740219 and No. 11165207 by the Ministry of Education, Science, and Culture, Japan.

[1] S. Iijima, Nature (London) 354, 56 (1991).

[2] H. W. Kroto et al., Nature (London) 318, 162 (1985); W. Krätschmer et al., Nature (London) 347, 354 (1990).

[3] S. Saito and A. Oshiyama, Phys. Rev. Lett. 66, 2637 (1991); M. B. Jost et al., Phys. Rev. B 44, 1966 (1991).

[4] N. Hamada, S. Sawada, and A. Oshiyama, Phys. Rev. Lett. 68, 1579 (1992).
[5] See, for instance, R. Saito, G. Dresselhaus, and M. S. Dresselhaus, Physical Properties of Carbon Nanotubes (Imperial College Press, London, 1998).

[6] For a review, see O. Gunnarsson, Rev. Mod. Phys. 69, 575 (1997).

[7] J. H. Schon, Ch. Kloc, and B. Batlogg, Nature (London) 408, 549 (2000).

[8] K. Suenaga et al., Science 278, 653 (1997); Y. Zhang et al., Chem. Phys. Lett. 279, 264 (1997).

[9] Ph. Kohler-Redlich et al., Chem. Phys. Lett. 310, 459 (1999).

[10] E. Bengu and L. D. Marks, Phys. Rev. Lett. 86, 2385 (2001).

[11] Y. Miyamoto et al., Phys. Rev. B 50, 4976 (1994).

[12] X. Blase et al., Europhys. Lett. 28, 335 (1994); Appl. Phys. A 68, 293 (1999).

[13] S. Okada et al., Phys. Rev. B 62, 9896 (2000).

[14] P.-M. Allermand et al., Science 253, 301 (1991), and references therein.

[15] For reviews, Magnetism I-IV, edited by G. Rado and H. Suhl (Academic, New York, 1966); E. Dagotto, Rev. Mod. Phys. 66, 763 (1994).

[16] T. Asada and K. Terakura, Phys. Rev. B 46, 13599 (1992), and references therein.

[17] E. H. Lieb, Phys. Rev. Lett. 62, 1201 (1989); 62, 1927 (1989).

[18] A. Mielke, J. Phys. A 24, L73 (1991); 24, 3311 (1991); 25, 4335 (1992).

[19] H. Tasaki, Phys. Rev. Lett. 69, 1608 (1992); Prog. Theor. Phys. 99, 489 (1998).

[20] M. Fujita et al., J. Phys. Soc. Jpn. 65, 1920 (1996); K. Nakada et al., Phys. Rev. B 54, 17954 (1996); Y. Miyamoto, K. Nakada, and M. Fujita, Phys. Rev. B 59, 9858 (1999).

[21] P. Hohenberg and W. Kohn, Phys. Rev. 136, B864 (1964).

[22] W. Kohn and L. J. Sham, Phys. Rev. 140, A1133 (1965).

[23] D. M. Ceperley and B. J. Alder, Phys. Rev. Lett. 45, 566 (1980).

[24] J. P. Perdew and A. Zunger, Phys. Rev. B 23, 5048 (1981).

[25] N. Troullier and J.L. Martins, Phys. Rev. B 43, 1993 (1991).

[26] L. Kleinman and D. M. Bylander, Phys. Rev. Lett. 48, 1425 (1982).

[27] O. Sugino and A. Oshiyama, Phys. Rev. Lett. 68, 1858 (1992). 